\title{
Eye Gaze Techniques for Human Computer Interaction: A Research Survey
}

\author{
Anjana Sharma \\ Research Scholar \\ Pawanesh Abrol \\ Associate Professor \\ Department of Computer Science \& IT, University of Jammu, J \& K, India
}

\begin{abstract}
Human Computer Interaction (HCI) is an emerging technology. Eye gaze technique is one of the very significant techniques of HCI and can be used as hands free pointing tool enabling hands-free operation of the display for the user. The important advantage in using eye gaze systems is that the user can communicate from a distance, and there is no requirement of physical contact with the computer. Investigation of eye gaze helps to understand various aspects of the user like attention, intention, desire and area of interest etc. The eye gaze detection techniques can be classified on the basis of direct eye detection, appearance, template, shape, feature, motion, hybrid, regression, 3D methods etc. There are significant factors like shape and size of the object, distance from the subject, texture, light conditions, colour, orientation, head movement, calibration which may influence and affect the efficiency and effectiveness of the eye gaze detection. The use of the gaze as a human computer interface in different fields is an example of high end applications of these techniques. Eye detection is being used in many real time and interactive high end applications. These include the tracking and analyzing of driver's behaviour with the head pose detection. It is being used for assessing consumer's shopping behaviour, pointing and selection, activating commands and combinations with other pointing devices, in surgical and medical applications. Moreover eye gaze techniques are also useful for designing and development of various devices especially for differently abled users.
\end{abstract}

In this paper an extensive research survey has been carried out to understand and analyze the study of various eye gaze techniques, algorithms and models. On the basis of survey of various techniques of eye gaze, a general overview of different phases of eye gaze processing has been presented. Certain technical factors have been identified that are significant and relevant for the working of the models. In the literature survey a number of parameters that are significant for estimation, detection, better efficiency and accuracy of eye gaze techniques have been studied and analysed. A comparison and analytical discussion of different eye gaze techniques and models have been presented. The analysis and classification of the models shall be helpful for further improvement and optimization in the performance and accuracy of eye gaze techniques.

\section{General Term}

Human computer interaction.

\section{Keywords}

Eye gaze techniques and models, feature based classification and comparison, phases, gaze detection and estimation.

\section{INTRODUCTION}

Eye movement-based human computer interaction (HCI) has become a very significant area of research in computer science. HCI refers to different techniques and algorithmic processes of non physical contact with the computing devices. These techniques are used to design, evaluate and implement various interactive computing systems.

These techniques help in developing and improving the user's interaction with the computer. A variety of engineering interfaces are being developed using these HCI based techniques [1-3]. HCI based systems are being developed for easy, effective, efficient, safe and enjoyable learning usage. These applications are of low cost and time saving. They provide convenience, security and safety to the users. The conventional input devices have certain limitations. These devices require the user's proximity and the physical touch making the system difficult to operate without user's hand. Moreover these cannot be used by differently abled people including those with some muscular disability. There are various types of $\mathrm{HCI}$ in which instructions to the computing systems can be given. These instructions can be given in different non conventional and non physical ways. Such approaches may require capturing and interpreting the face, motion of head, eye gaze, hand, arms or even the whole body. Such techniques use detection, analysis and recognition of facial expression, hand gestures, head pose, emotion, speech or voice, eye gaze etc. for further processing. Analysis of facial expressions like happiness, hatred, fear, sadness, disgust, anger and surprise may help in understanding and improving the human interaction and communication with computing systems. Face detection techniques include the extraction of facial features like forehead, chin, eyebrows, eyes, nose, mouth and hair line etc. Especially eye localization algorithm also help in accurate face recognition. Face detection techniques are very significant in fields like image processing, pattern recognition and authentication and also help in interactive and real time applications of computer vision $[4,5]$. Hand gesture based methods use the hand, as an input device without any physical touch. These methods can be utilized for navigation, selection, and manipulation tasks in virtual environments (VE) such as surgical simulations and training systems. Electro-mechanical or magnetic sensing devices like data gloves are some of the effective tools for detecting hand movements. However there are certain difficulties. These include high-dimensional problem, self-occlusions, processing speed, uncontrolled environments, and rapid hand motion etc. in these methods [6]. Head pose is estimated by fitting result of whole-face model. Head motion can be categorized into different movements like parallel to the image plane, parallel to the optical axis of the lens and rotations, turning right or left, facing up or down, neck declining [7, 8]. Emotion recognition helps in finding out the real emotion or feelings of the user like anger, happiness, sadness etc. Voice or speech recognition is helpful for identification and authorization of users. Voice recognition can be used by the pilots, radiologists and users for hotel and railways reservation. The noise level, noise type, position of the microphone, and speed and manner of the user's speech are some factors that may affect the quality of speech recognition. These methods also have certain limitations like inaccuracy, low audio quality etc. Eye movement and detection is an image processing technique to locate eye 
position from an input facial image. However more intuitive and natural human interfaces can be developed with certain improvements in eye gaze techniques.

The eye detection is widely used in face detection and different areas like artificial intelligence (AI), virtual reality (VR), ubiquitous computing (UC), augmented reality (AR), artificial neural network (ANN) etc. Eye gaze is another method of HCI. In such techniques real time data is gathered for tracking and estimation of eye gaze. This estimation is generally done in relation to gaze direction of the eye position and movements. It is further used for executing desired instructions by the user to the computing system for investigation, analysis of the desire and emotional state of the user. HCI applications like eye detection are being used for analyzing driver's behaviour with the head pose detection [9], in designing simulators for surgical and medical applications [10], for capturing and controlling brain signals as a Brain Computer Interface (BCI) [11]. HCI techniques are also being used to build gaze control strategy for controlling different devices like microscopes with eye, GUI based eye gaze application control, estimating user's interaction in the simulated environment and developing simulator for computer games etc. Certain FPGA (Field Programmable Gate Array) models are also being further used for designing FPGA based sensors, tracking sensors and other embedded devices. Real-time systems for iris recognition, eye contact correction in video conferencing etc. also use eye detection techniques [12]. Some other high end applications of eye gaze techniques include detection of sleepiness in drivers and for improvement in web usability [13]. The main functions of eye gazing systems are the human activity recognition, facial expression recognition, pointing and selection, activating commands and combinations with other pointing devices. Similarly the outcomes from the eye trackers or models can also be used for capturing and controlling brain signals as a BCI [11], eye gaze interfaces using EOG [14], gaze tracking system for children [15]. Eye gaze based techniques can also be used to increase security or authenticity of input codes for financial transactions etc. Eye gaze patterns are being investigated for detecting lie or for recognizing a specific person or scene [16]. Assessment of the impact of consumer shopping behavior, packaging design and product placement, developmental psychology, sports, ophthalmology, neuroscience, nonhuman primate research, psycholinguistics and advertising research are among the many fields where the eye gaze techniques are being explored. Eye gaze techniques may enable the differently abled people to use the computing systems more easily and comfortably. This method of interaction is a unique and effective tool for disabled individuals like mentally retarded, differently abled and old aged people where eye movements may be the only available means of communication and interaction with the computer and other people [17, 18]. This technique is also helpful for those who are unable to hold or operate the keyboard or mouse due to some muscular or other abnormalities. Different methods for estimating and detecting eye gaze are discussed in the next section.

\section{EYE GAZE TECHNIQUES}

The eye gazing is the process of measuring either the point of gaze or the motion of an eye relative to the head. The gaze point is estimated after acquiring the movement of the eye [19]. It requires significant levels of accuracy and estimation so that certain desired instructions can be executed by the computing system. Eye gaze techniques require a number of eye inputs which can be taken by a capturing device like a digital camera, a webcam, single or multiple cameras etc. The eye inputs may be the contour of the visible eyeball region, intensity distribution of the pupil(s), iris and cornea, as well as their shapes. There are various features of the eye that can be analysed for ascertaining the eye gaze. These features include the location of pupil in the visible eye area, colour, texture, light conditions, the position of the iris within the eye socket, state of the eye related events like single eye blink, double eyes blink and other blink combinations, one or both eyes shut. Besides some more attributes like frowning of eye, eye ball movements, view or visual angle, gaze time etc. may help for further analysis. For the eye gaze estimation and detection, a number of hardware devices, software, lighting conditions and different flexible setups are required. Many of the current methodologies use different devices or light sources to follow the eye gaze. The process of eye gaze involves the user to look at the specific location on the screen. Eye gaze techniques include all the algorithms, models and methods including eye tracking. The broad process of eye tracking system can be explained as follows. Initially the data related to the position of the eye is acquired using a system that illuminates the eye of a subject with an infrared (IR) source. It acquires a video image of that eye and locates the corneal reflection (CR) of the IR source. It is processed in real time to calculate or records the eye gaze along with direction. Once the pupil has been detected, the corneal reflection is determined from the dark pupil image. The reflection is then used to estimate the user's point of gaze in terms of the screen coordinates where the user is looking at. An initial calibration procedure may also be done by the commercial eye trackers.

The gaze camera extracts the center of the pupil, and the gaze direction is estimated from their coordinates. The different actions can be recorded based on different eye inputs like blinking, frowning of eye, eye ball movements, view or visual angle etc. [20]. Different eye tracking calibrated or non calibrated hardware devices like eye trackers, gaze trackers or head mounted devices which may provide sufficient information. Raw data from an eye tracker cannot be directly used for gaze-based interaction. This is due to the presence of noise from image processing or removal of distorted objects. The image thus obtained may further be normalized by performing different kinds of preprocessing like denoising, reducing the automatic white balance from the cameras, adjustment of colour tone, gray scale, identification etc. This image is then further analysed for identification for various parameters. This information may be used by gaze based applications for moving the cursor on the screen.

The eye detection techniques can be classified on the basis of direct eye detection, appearance, template, shape and feature, motion or hybrid methods. The direct eye-detection methods search for the eyes without any previous information about face location. It can further be classified into passive and active eye detector methods. Passive eye detectors work on natural images, without any special illumination. It can, therefore, be applied to movies and for news broadcast etc. Active eye-detection methods use special illumination and retro-reflection property of the eye. These methods are applicable to real-time situations in controlled environments like eye-gaze tracking, iris recognition, and video conferencing [12]. The appearance based methods also known as image template or holistic methods detect and track eyes directly based on their photometric appearance. These methods use models built directly on the eye region appearance. These methods also take into consideration the colour distribution or filter responses of the eye and its surroundings. Such methods can use the shape of the eye, eyelids, eyelashes as well as pupil, iris and glints and these should be accurately detected to obtain an accurate estimation [15, 17]. Appearance based methods usually collect a large amount of 
training data with the eyes of different subjects, under different face positions and illumination conditions.

In the template-based methods, a common eye model is first designed and the template matching is then used to detect the eyes on the image. The deformable template method is a commonly used template based method. In this method the eye position is detected via a recursive process. This method can detect the eye accurately, but it is computationally expensive, demanding and high contrast image is required [21-23]. In principle, the template matching technique is weak in pattern rotations and scale changes. The template 'Between the Eyes' is also used for tracking of eyeposition tracking. These are further updated frame-by-frame based on the current located eye positions. Eyes are searched based on the current position of 'Between-the-Eyes' and their geometrical relations to the position in the previous frame. This template has good features for accurate tracking and is observable for a wide range of face orientations [8].

The shape based methods are developed either from the local features like limbus, pupil, eye corners or from their contours. These methods use a prior model of eye shape and surrounding structures.

These methods use already developed models of eye shape and surrounding structures. The feature based methods use the characteristics of the eye to recognize the distinctive features like limbus, pupil or cornea reflection around the eyes [5, 12, 24]. These methods identify only the local features of the eye and face and are less sensitive to variations in illumination as compared to the appearance based method. Feature based methods usually require a considerably smaller amount of calibration samples than appearance based methods [15].

Another method using the eye motion patterns also called eye gestures can be used to detect the eye movements. This can be classified as eye contact, deictic gestures and non-deictic gestures. Eye contact implies one participant looking at the other participant and the listener usually interacts with the speaker with fairly long gazes. Deictic gestures are eye gestures with a specific reference to an uninvolved person or an object. Non deictic gestures or gaze averting are eye movements to empty or uninformative regions of space [25]. Hybrid methods combines the advantages of different eye models like colours and shape, shape and intensity etc. based on features, appearance, shape etc. to overcome their shortcomings [17]. On the basis of physical contacts eye detection techniques may use intrusive, non intrusive or remote, diagnostic and interactive trackers. Intrusive trackers include physical contact with the user. In this type of trackers the eye gaze estimation is done on the basis of the voltage induced in the embedded scleral search coils by an electromagnetic field [26]. If the coil is attached to the eye, then a signal of eye position will be produced. Although it is an invasive method and invaluable research tool but it require something to be placed into the eye and is rarely used clinically [4]. Another estimation of eye gaze technique uses electrooculography (EOG) for detecting eye movement by measuring small differences in the skin potential of the retina [14]. Remote trackers use cameras to capture image of the eye. These are suitable and acceptable in virtual environments and AR [13]. Infra-Red Oculography (IOG) is another technique of remote tracking which is invisible to the eye. A fixed light source is directed at the eye and the amount of light reflected back to a fixed detector will vary with the eye's position $[10,15,27-30]$. It has been used in a number of commercially available eye trackers.

Diagnostic eye trackers records the viewer's PoG in an objective and quantitative method and this information helps in examining people while watching commercials, using instruments in plane cockpit. These trackers also help in analysis and understanding of human attention and desire, interaction with user interfaces. The interactive tracker consists of the eye positioning unit detects the user's eye position whereas the gaze tracking unit to detect the user's gaze direction from the eye image taken by a near-infrared camera. The gaze tracking unit is placed on a pan-tilt stand so it can change its direction to detect the user's eye. The interactive eye trackers react to the user's gaze either as a control input or as the basis of gaze-contingent where the system adapts its behaviour based on visual gaze or attention of the user [17]

Another method used for eye detection is based on linear and non linear filtering. Non linear filtering method gives better performance in detecting the eyes than traditional linear filtering approaches. These methods detect eye corners [24]. Certain systems use 'purkinje images' for eye tracking. These images are reflections of the light source from various surfaces in the eye. The first Purkinje image or corneal reflection is often referred to as the glint. These images give an eye position signal $[27,28]$. Different methods that automatically extract the eye position from images of the eye have been developed with the help of video and image analysis. These gaze detection methods employ Haar-like features for object recognition [30] and neural networks to classify the gaze direction in real time. Support vector machines (SVM) are also used in eye detection system to estimate a mapping between the appearance of the eyes and the corresponding gaze direction. These determine the presence or absence of eyes using an input vector consisting of gray values in a moving window [15]. Certain methods also use template matching, eigenspace, and integral projection. In template matching face recognition is parameterized by using a function for a standard frontal face pattern. The existence of face is determined with the help of sub templates and the correlation values. These values are computed for the face contour, eyes, nose and mouth independently [4]. In principle, the template matching technique is weak in pattern rotations and scale changes [8]. In eigenspace the detection of the eyes, nose and mouth is done by a hierarchical search in scale and space for a region in the image. This projection matches the signature of the feature to be detected. The eigenspace method shows better performance than the template matching method, but its performance is largely dependent on the choice of training database set $[4,32]$.

A different method which is also used for locating the eyes is Gabor filters. It is used to locate features of the eyes including the corners of the eyes and the iris. These are used to detect and track the corners of the eyes as well as to find the iris. A particular edge is being found out firstly for e.g. left corner of the iris by a sequential search and then using the same course, filters are used to track the edge of the iris. Further in the same manner the corners of the eyes are being found out. The accuracy rate of this method is $91.6 \%$ for the inner eye corners [33]. The non linear filtering method is superior to Gabor wavelet-based linear filtering method which is having energy localization property in both the space and frequency domains.

The gaze-tracking is categorized into two types of methods remote and head-mounted. The remote mounted normally uses number of cameras, such as narrow-view and wide-view cameras or stereo cameras with multiple illuminators. These are high cost system with a high level of complexity due to the use of a two-camera system. Complicated calibrations are also required which are difficult for the general user but are accurate and convenient. The head-mounted gaze-tracking method is less convenient than the remote gaze-tracking method. This method calculates the gaze 
position by using a wearable head-mounted device, including a camera and near infrared (NIR) illuminators. These systems are often incorporated into glasses or headsets [26, 34].

The gaze estimation technique can be regression based and $3 \mathrm{D}$ based models. The regression based methods are simple and accurate and use a single glint and are suited for particular applications with the restricted head movement.

The 3D based model eye tracking systems require one time system and geometric calibration with natural head movements [4, 25, 35]. Although the 3D-based method is more accurate than the 2D-based one, the former requires much more processing time and requires complicated calibrations between the camera and monitor, which makes it difficult to be used for large-sized display interfaces [34].

There are various kinds of eye tracking systems which are built upon eye gazing techniques available in the market namely SMI, ASL, LG, Tobii etc.

Most of the commercial and existing eye-tracking systems measures point of gaze (PoG) by the 'Pupil-Centre-CorneaReflection' (PCCR) technique.

In the developmental psychology, eye tracking helps to explain growth and transformation in perceptual, cognitive, and social abilities from infancy through young adulthood. In psycholinguistics linguistic researchers investigate human language development, language skills, and reading behavior. In the field of ophthalmology, eye tracking is used to study oculomotor behavior, visual functions and vision deficiencies. It can also help in assessing the impact of consumer shopping behavior based on real attraction of the consumers, packaging design and product placement. The eye tracking research can be used by integrating eye tracking cameras into automobiles to assess in real time the visual behaviour of the driver [9]. Further eye tracking can be used for analyzing preferred reading regions on a monitor [36]. Further eye track tracking is also being explored for the neurological related diseases. Eye gaze study of non human primates helps to assess the behaviour of human beings. A lot of research work has been done in this field. A detailed and comprehensive literature review of different proposed eye gaze models and algorithms is presented in the next section.

\section{LITERATURE REVIEW}

In this research survey, various algorithms, models and techniques have been studied. On the basis of survey certain significant parameters and factors that affect the accuracy and efficiency of eye gaze techniques have been identified. Various eye gaze based HCI models and algorithms have been studied for further analysis and investigation. A discussion of the merits and demerits of eye gaze based models has been presented below.

Early work on interactive eye tracking applications was based primarily on users with disabilities but can also be used for general users dealing with portable computers [17, 19].

Hewett et al. have emphasized the contents, application areas and need for various techniques in HCI [1]. In another paper the researcher's P. Corcoron et al. focused on the point that real time face detection combined with eye gaze tracking can provide a better means of user input into a gaming environment. This information can be used in various ways to enhance the user interface design and models that are sensitive to a user's behaviors and mood. Further they stated that eye-gaze can be used as a means of direct user input for improving the accuracy of eye estimation. Moreover they have given the solutions to enable real time human face detection and tracking with a good algorithm for eye tracking. The proposed system does not require any wearable attachments, supplementary lighting. There is no use of the eye-glint phenomena. It only employs a single user-facing camera. The accurate measure of the eye gaze is done by the edge corners, center of the eye socket and the eye pupil [5].

K. Kinoshita et al. developed a fast and robust head pose and gaze estimation system which can detect facial points and estimate 3D pose angles and gaze direction under various conditions. Only one face image is used as input without any special devices such as blinking LEDs or stereo cameras. Without calibration the system shows a 95\% head pose estimation accuracy and $81 \%$ gaze estimation accuracy. The proposed system used 3D shape model for estimating head poses and gaze information [7]. There are many applications and fields where eye gaze techniques can be helpful like in car driver's behaviour [9], medical field [10] etc. is discussed in the next section.

The proposed method uses a real-time contact free eye-gaze tracking system and approached the problem of gaze detection for tracking the gaze of a person while driving a car without disturbing the driver. It can be used for many other applications like in medical field or even to build human computer interfaces for handicapped people. The system processes in real time 25 frames per second for images taken by the eye camera. It allows gaze tracking for vision angles over $+45^{\circ}$. Estimation and searching of pupil and iris centre and contour is done in the proposed algorithm. The main objective of this work has been building a non-intrusive eye-gaze tracking system. The proposed algorithm determines the pupil centre with sub-pixel resolution. The measurement error in the pupil-glint distance which is easy to detect and track is minimized by A. Perez et al. [9].

For medical and surgical purposes M. Wilson et al. proposed a model to examine gaze strategy and eye hand coordination to differentiate between the working of experienced and novice operators. The observation was done with a two handed surgical task on a VR laparoscopic surgical simulator. Different parameters like performance, movement, gaze, and eye-hand coordination were recorded and compared. Further it has been found that the experienced surgeons completed the task significantly more quickly with fewer movements and displayed shorter tool paths. The finding of the study is further used for the utility of examining strategic gaze behaviour and eye-hand coordination measures of experienced surgeons. The proposed gaze strategy revealed that experts generally used a target-focused gaze strategy during the task without focusing much on the tool position as compared to novices who focuses equally on both target and tool locations [10] The researcher's in the next review presented a hardware FPGA based embedded system for eye detection with a single-chip eye gaze tracking sensor.

A hardware algorithm for fast eye pupil candidate detection is given by Amir et al. The researcher's presents a hardware-based embedded system for eye detection, simple logic gates, with no CPU and no addressable frame buffers. For exploiting the hardware implementations the paper presents a prototype of a system which uses a CMOS digital imaging sensor and an FPGA for the image processing. The researchers highlighted that the data transfer bandwidth and sequential data processing rate are the two main flaws of any CPU-based real time image-processing algorithm which makes the existing CPU to do extra actual computation. The algorithm's output is a list of pupil location and size. The proposed algorithm can further be extended to detect glints by integrating into imaging sensors VLSI. This approach can solve the speed, size, complexity and cost of current eye-gaze tracking systems to a 
certain extent. However a major drawback of the proposed hardware solution is that the subjects cannot blink and they can't move their head. The system requires very sensitive sensors and this limits the distance range of eye detection in the proposed system. Adding the detection of cornea glints to the pupil detector make it a single-chip eye-gaze tracking sensor [12]. Another not so common eye gazing technique called EOG has been used by the authors for recording eye movements is discussed in the next review. In the paper by T. Yagi the author used another method of eye gaze technique, the electro-oculography (EOG) and developed two types of eye-gaze interfaces namely EOG Pointer and EOG Switch. EOG pointer enables a user to move a computer cursor or to control a machine using only eye-gaze while an EOG switch output an ON/OFF signal only. Various recording eye gaze interfaces have been developed by the researchers since 1980 . These are infrared oculography (IROG), the limbus tracker and video oculography (VOG). These interfaces have time-consuming calibration, no stability against blinking and relatively unaffordable. In order to overcome these disadvantages, another eye-movement recording technique, EOG which allows the standing potential between the cornea and the posterior pole of the eye to be recorded is proposed. This technique can be used with less fatigue with long recording, can be easily maintained and is of low cost. However, blinking and movement of facial muscles create serious noise issues. EOG has not become common because of the drifting of the EOG signal's baseline due to DC amplifier. An algorithm is developed to calibrate the drifting signal and a technique is also developed to reduce the blinking effect in the proposed paper. The disadvantages of EOG can be solved with the use of adequate signal processing, calibration, and the choice of an amplifier [14]. The eye gaze tracking system can be used for animals and infants of one year of age for studying their visual behaviour with offline calibration as proposed by the researcher's discussed in the next paper.

A head-mounted gaze tracking system for adults, animals and infants of one year of age is presented by B. Noris et al. for the study of visual behavior with two CCD cameras that allows studying both the central and peripheral vision with offline calibration. Illumination and normalization is used to increase the working of the system and eye blinking detection to avoid tracking errors. SVR is used to estimate a mapping between the appearance of the eyes and the corresponding gaze direction. The system can be used successfully indoors as well as outdoors with less performance in outdoors and reaches an accuracy of $1.59 \mathrm{o}$ with adults and 2.42o with children. A wearable device WearCam is proposed by combining two wide angle cameras. Moreover the intrusiveness of the system is very low. Most of the gaze tracking systems focuses only on central vision whereas the proposed system uses to study both central and peripheral vision at the same time. The system design does not hinder or distract the wearer's field of view, as no elements are placed directly in front of the eyes. The underdeveloped eye parts like eye lashes or eyebrows and lack of impatience in children results in fewer amounts of image details. Further, the proposed gaze tracking system has wide range that allows the study of the gaze behavior in the whole field of view as compared to the previous restricted range [15]. A review of several models, methods, algorithms have been given by the authors in the next section.

A review of different eye gaze techniques, models and tracking along with gaze estimation methods has been presented by D.W. Hansen et al. A comparative analysis of different methods and models has been presented. The paper suggests that the future gaze tracking systems should be of low cost, easy to setup, minimal or no calibration, less use of IR and good gaze estimation accuracy under varying illumination conditions and natural head movements. The authors further observe that various factors like distance of the subject to the camera, age related intrinsic property, orientation, illumination effect the accuracy of the eye gaze techniques. The eye detection techniques discussed in this paper consists of shapebased, appearance-based feature based and hybrid methods. Many existing eye trackers are based on active light schemes which are particularly efficient indoors and in dim environments [17].

DASHER, a new kind of dictionary which uses eye gaze based direction is developed by D. Ward and D. Mackay. This technique is friendly, accurate and faster. In this system, letters are presented vertically in alphabetical order on the right side of the screen and the user gazes at the desired letter. The letter moves towards the centre while the interface zooms. Gazing right and left on the screen leads to a selection of the target and deletion of written text. In comparison to an on-screen keyboard the Dasher users could write at up to 25 words per minute. The error rate with the onscreen keyboard was about five times that obtained with Dasher. It can work in most language and adapts to the user's language. The authors assert that it can also be operated with a touch screen or roller ball. However, the zooming technique allows low accuracy eye tracking [18].

In the research work by K.N. Kim et al., the direction of eye gaze with head movement is used for the investigation. The exact eye position is detected with the help of two proposed algorithms for iris center detection namely the longest line scanning (LLS) and occluded circular edge matching (OCEM) algorithms. The main stress by the authors is given on the eye movement, not on face and eye location. The geometry based estimation is better than adaptive estimation in estimating eye-gaze. A small number of failures are reported due to the errors in large head movements and error in eye movement tracking. Also the camera resolution limits measurement of eye movement as observed by the authors [19].

Another proposed method using low cost webcam with no ANN, no calibration is being discussed in the next review. Two shape and intensity-based deformable eye pupil-center detection and movement decision algorithms are introduced by I. F. Ince et al. A low cost system for 2D gaze estimation and cursor control system regardless of the real position of pupil-center is presented in this paper. One algorithm is for the detection of the eye-ball with stable approximate pupil-center and the other one is for the eye movement direction detection. Eyeball is detected using deformable angular integral search by minimum intensity (DAISMI) algorithm. Deformable template based 2D gaze estimation (DTBGE) algorithm is employed as a noise filter for deciding the stable movement decisions. While DTBGE employs binary images, the DAISMI algorithm employs gray-scale images and searches the densest dark contour or shape in an eye estimate and tracks the possible eyeball contours in an angular way. It has been observed by the researchers that every single eye gaze tracking system has different visual angle accuracies which are generally used for benchmarking of the eye gaze systems. The proposed system has no calibration, no training process, no infrared filtering with head mounted camera, no complex artificial neural network and it is the only system which does not need real pupil-center position for 2D gaze estimation. This system is an ideal, better and cheap option for those who cannot afford expensive trackers, does not use calibration, complex ANN. Better accuracy has been achieved with a simple webcam with higher success rates [23]. Further experiments using the linear and non linear approaches for eye detection have been done by the researcher's. 
Two methods have been proposed for eye detection based on linear, nonlinear filtering and face modeling by Sirohey et al. Edges of the eye's sclera is detected with four Gabor wavelets. The linear filtering method is applied to detect the eyes. However the authors report some false alarms in the result, although the detection rate was good. A nonlinear filtering method was therefore developed to detect the corners of the eyes in the color image of the face. The result indicates a better detection rate using this method. This method gave a $90 \%$ detection rate with no false alarms. High quality images are essential for this method and the algorithms have significantly less complexity. The property of 'eyeness' has been considered during the experimentation. The non-linear filtering method had a higher level of performance in detecting the eyes than the linear filtering approaches. Based on selection of proper threshold the detection and false alarm rates can be improved [24]. Further techniques have been developed by the authors for reducing difficulties in calibration procedures by using eye ball models, purkinje and pupil image processing as discussed below.

A novel gaze tracking system called FreeGaze method implies a few geometric and image processing with a single light source using a calibrated camera and single glint. The setup reduces the problems faced while using complicated and difficult calibration procedures. The proposed system needs only two points on the screen for each individual calibration. FreeGaze detects gaze position by the two processes. First, the pupil and the purkinje image are detected from the captured image. Then the gaze position is computed accurately from these two images by using the proposed eyeball model by the researcher's T. Ohno et al. However if the gaze at the point on the screen is not accurate, there can be an error in the calibrated data. Further the detection of the accuracy of the personal calibration was insufficient [25]. Later, the same authors proposed in another paper that personal calibration can be reduced to two fixation points using two light sources and a single camera as discussed further.

Most of the gaze tracking systems perform calibrating experiments before using the systems and are dependent on certain parameters of the user like radius of the cornea, the position of the pupil, head position. However S.W. Shih et al. in their paper proposed calibration free gazing method that employs multiple cameras and multiple spotlights to estimate the light of sight (LoS) without using any of the above parameters. The method compensates the refraction on the surface of the cornea. It uses the purkinje image to determine the location of the eye. The estimated 3-D position of the eye and the observed centers of the pupil images are then used to compute the eye gaze direction using a safe and cost effective infrared LED as the point of light source. In spite of the correction of the refraction on the surface of cornea some measurement error remains in the experiment if there is no calibration [28].

J. S. Agustin also presented a low-cost gaze tracking system that is based on a webcam mounted close to the user's eye. The performance of the gaze tracker is evaluated in an eye-typing task using two different typing applications. Further, the provision for open source solutions and the software contribute to fit specific needs. An inbuilt ITU Gaze Tracker is used and an evaluation of a head mounted setup is presented which uses a webcam with builtin infrared light and is very easy to build. The evaluation is by using GazeTalk and StarGazer. The system offers a high flexibility and demonstrates that a gaze tracking system built from inexpensive components can also be robust and accurate. Lack of head-pose invariance is the main drawback of low cost webcam. However, with the reduced size and weight of future webcams make it possible to mount them firmly on eyeglass frames [29].
The proposed method in the paper is a refinement of the previous work [26] by the same authors. A free head gaze tracking system is described with simple personal calibration without any kind of head gear. Personal calibration is done by looking at the two markers on the screen. The accuracy of the implemented system is about 1.0 degrees. The basic strategy of the method is to estimate the position of center of the pupil and center of the corneal curvature with high accuracy. The reduction in the acceptable eye area is a limitation in the eye positioning unit but it works better than the standalone eye positioning system [30].

Another gaze estimation method has a large-sized display at a distance which calculates a user's gaze position by using a wearable head-mounted device, including a camera and near infrared (NIR) illuminators is proposed by C. Choo et al. The effectiveness of the presented approach is tested with $3 \mathrm{D}$ VR system, which is implemented by a VR modeling language. The gaze-tracking accuracy is not affected by head movement since the proposed method uses a near infrared camera to track the head. The accurate gaze position is calculated by using two support vector regressions (SVR). The head-mounted gaze tracking method used by the researcher's because of its convenience, system cost, complexity, calibration, and accuracy beside the fact that it is less convenient. They have used 2D-based method for the research as the 3D-based method require more processing time and complicated calibrations which are not suitable for large sized display interfaces. The gaze estimation method for large-sized display based on a light glasses frame is proposed in the paper to overcome the problems of the previous studies. The experiment results showed that the RMS error of gaze estimation was $0.79^{\circ}$ for a 30-inch screen [34].

In the next paper the authors has brought out the two characteristics of the available remote eye gaze trackers using pupil center cornea reflection (PCCR). A convenient natural head movement and minimal calibration procedure is proposed only one time for a new user. A different simple 3-D gaze tracking technique is proposed which can estimate the optic axis of the eye without knowing any eye ball user-dependent parameters. The 3-D gaze direction can be estimated in a more easy way, improving the robustness and accuracy of the gaze estimation. A novel 2-D mapping-based gaze estimation technique is introduced for free head movements. The average horizontal angular gaze accuracy is 1.47 and the average vertical angular gaze accuracy is 1.87 , acceptable for many HCI applications. In another experiment, the effect of the distance to the camera on the gaze accuracy is analyzed. The results show that as the user moves away from the camera, the gaze resolution will decrease. The 2-D mapping based gaze estimation technique used by the researcher's is more accurate than the 3-D gaze tracking technique. The proposed methods by Z. Zhu et al. will improve the usability of the eye gaze tracking technology. By improving the calibration accuracy for the 3-D gaze estimation accuracy the source of errors for the 3-D method can be improved [35]. Improved eye gaze models find their applications in variety of areas.

The slow text entry of the system is improved by the researchers in the next paper.

Another research work done by Linda. E. Sibert et al. investigated experimentally the strength and weaknesses of the eye gaze techniques. They have used fixation recognition algorithms for comparison of the two techniques namely mouse based and eye gaze based. Eye gaze based interfaces utilize natural eye movements and thus are fast, easy and intuitive [37]. 
An alternative to DASHER [18] has been proposed by M. Urbina et al. The authors highlight the single text entry and the longer dwell time that are the main drawbacks of the proposed DASHER system. These drawbacks are overcome by using already developed typing interface based on hierarchical pie menus pEYEwrite. The proposed method achieves text entry speeds of about 13 wpm by combining different selection methods with bigram entry and word prediction. An important limitation of the proposed system is that the use of pEYEwrite combined with bigram entry repeats entry of double errors [38].

Another proposed method does not require special calibration action of users in contrast to the conventional methods which requires looking at several reference points in the calibration process. The method can be realized by combining 3D eye-modelbased gaze estimation and circle-based algorithms for eye-model calibration. A single-camera-based gaze estimation algorithm is proposed that does not require the attachment of any devices or markers for gaze estimation or such special user action as looking at reference points in the calibration. The proposed method by $\mathrm{H}$. Yamazoe et al. can be applied to different applications as gazebased interactive signboards, communication evocation using gaze motion etc [39].

An intelligent tutoring system (ITS) first of its kind has been developed by S. D. Mello et al. for detecting student boredom and disconnection with the help of a eye gaze tracker. The tracker monitors students' gaze patterns for identifying his interest or attention in the subject. The proposed system reorients the attentional patterns and attempts to reengage the student with different dialog moves. The evaluation of the proposed system indicates the promotion in learning and motivation in the students. The gaze reactive ITS can also be used in the classrooms as better and effective teaching aid [40].

C. Hennessey et al. introduced a non-contact eye tracking system that allows the viewer's movement in a big room to make HCI with latest Smart TV's or other large displays more effective. The longrange eye tracking system hardware includes a pan and tilt servo mechanism, a wide-angle camera, and an eye tracker with a narrow-angle camera [41].

The paper by J.G. Wang et al. is in contrast to the earlier researches which are based on the research on the two eyes details and the work on images with the full face. This makes the eye details small and accuracy is affected. The main contributions of this paper are to show that it is possible to estimate eye gaze with only one eye image and that consequently this achieves higher accuracy of eye gaze estimation. Morphological image processing operations like open, histogram are also used for accurate gaze determination. The proposed method is non-invasive, fast and robust because the segmentation of the iris contour is one of the simplest and most robust facial features to extract [42].

N. A. Villani et al. reviewed the work of other researchers on the eye gaze movements like saccades which are lines indicating the paths that the eyes took

across the screen and fixations which are circles that show the locations at which the eyes dwelled on the screen for a length of time above a specified threshold. The 3D model is characterized by 30 parameters like pupil size, lens, eye motions, eyelid motions and camera motions. This is the most advanced and physiologically accurate eye simulator available for applications to medical education, character animation and HCI as concluded by the researchers [43].
There are major research issues highlighted by various researchers in the future gaze tracking systems. The focus is on the use of low cost systems, optimal use of available resources, using existing available resources, maximizing the extent of visual angle, reducing the complexity of the algorithms. The reduction in the need of cumbersome training of subject and equipment is required. The intrusive trackers despite being accurate are useless due to their intrusive nature in most of the computer graphics and imaging applications. Minimizing resource utilization should be taken into consideration. Minimal or no calibration, less use of infra red light source and good gaze estimation accuracy under varying illumination conditions and natural head movements are also required. Detecting the eye movements is not difficult with restricted head movement. The eye movements are difficult to detect when the head is moving because of the unwanted features around the head boundaries, the eyebrows, nose, mouth, and other parts of the face. Further there is a need of a gaze tracking system that will have a distant camera and starts gaze detection as and when required to strongly improve the above mentioned research issues. These issues may further be improved in order to accept the eye gaze technique as a natural computer input device. The estimated gaze should have high level of accuracy. The detected regions should only have the required regions of the eye only. Proper identification of saccades and fixation are required. The issues related to the undoing of the previous action as a limitation in DASHER should be taken care properly. The current and existing commercial and research systems require a dedicated computer for the image processing task and are generally large, expensive, and complicated-to-use. In order to make eye-gaze tracking ubiquitous, the system's complexity, size, and price must be reduced to a lower extent.

The accurate detection and estimation of the pupil or cornea of the subject is one of the significant factors

for the eye gaze models. This factor further depends on the distance of the subject to the camera, age related intrinsic property, orientation, illumination effect the accuracy of the eye gaze techniques. Eye

gaze analysis models may use different statistical and probabilistic models like principal component analysis (PCA) used to detect the user's eye position from the stereo camera output, correlation and regression.

Further it has been observed that to enhance and improve the working of the eye gaze techniques, most of the existing devices and techniques should use specific devices and light sources for eye gaze estimation. Infrared illumination is used for easier tracking. In spite of being accurate these devices are costly, complex and unaffordable. To increase the reliability of the recorded data, eye tracking technology needs to be improved. The captured data should be robust and accurate so that the reliability of the recorded data can be improved and the point-of-regard measurement remains accurate without the need for frequent recalibration. The gaze tracking framework should be userindependent, non-intrusive, automatic initialized and robust to eye glasses. Lot of research has been done on the basis of different models or methods like shape, appearance, feature, template, hybrid, regression and 3D based models. Different types of trackers like head mounted or remote mounted are being used for detecting eye movements. Different types of features of eye like pupil, glint, purkinje image, iris and cornea etc. have taken into consideration for estimation and detection of eye gaze. In order to improve the eye gaze models, systems and devices. There is a need to understand certain significant factors like shape and size of the object, distance from the subject, texture, light conditions, colour, 
orientation, head movement, calibration, environmental set up etc. The efficiency and effectiveness of the eye gaze detection depends on these factors and therefore have to be analysed. Out of these factors we have selected certain important factors and analysed few selected models of eye gaze models on the basis of selected parameters. A comprehensive result and discussion of the analysis of the selected models have been presented in the next section.

\section{RESULT AND DISCUSSION}

On the basis of comprehensive and extensive research survey as discussed above, an understanding of various phases of processing of eye gaze techniques, their classification and various factors that influence the working and outcome of the results have been studied and analysed. The outcome of this research survey is being presented in two following headings:-

1) Overview of eye gaze processing.

2) Feature based comparison of different eye gaze techniques.

\subsection{Overview of eye gaze processing}

After the extensive research survey and literature review of existing eye gaze techniques, it has been observed that there are specific phases for the eye gaze processing as shown in Figure 1. Each phase is a set of one or more logical procedures that may be of colour tone, gray scale, identification and removal of distorted objects etc.

Initially, an image is captured with the help of an image capturing device C. It may be a normal camera, webcam, single or multiple cameras etc. required to perform certain functions on the input signal. These functions may be denoising, adjustment The capturing device may capture the image of the eye of the subject placed in the background B. Both $\mathrm{C}$ and the subject are placed in the working area $\mathrm{W}$.

The output Ir from this phase is further processed in the second phase. Infrared LED's light sources may also be used.

In the next step of illumination, acquisition and normalization, the issues related to setting up of the capturing device and computing system, image formats, issues of compression, online and offline

acquisition or retrieval may be considered to improve the process of image capturing.

Further the size of the raw image thus obtained may further be normalized for doing some meaningful processes and obtaining specific results. Next phase is preprocessing. The digital image may further be standardized by performing different kinds of preprocessing like denoising, adjustment of colour tone, gray scale, identification and removal of distorted objects. The image thus obtained is then analysed for identification for various parameters.

For this, various eye gaze estimation and detection algorithms may be applied. These may include DTBGE and DAISMI, PCCR, eye ball model, dasher, linear filtering method etc. Such algorithms can use $2 \mathrm{D}$ or $3 \mathrm{D}$ estimation technique. These models utilize one or more of the following features like corneal detection, glint detection, purkinje image, refraction at the surface of the cornea and high order approximation etc. The results from the outcome phase can be used for further fetching and execution of the instruction correctly.

The accuracy of the eye gaze models depends on the other factors like visual angle, determination of iris radius, center of the pupil and center of the cornea, distance between pupil and cornea, edge corners of the eye region, the center of the eye socket and the center of the eye pupil, distance of the subject from the camera, pupil size, lens, eye motions, eyelid motions, camera motions etc. Careful selection of the parameters, controlling and adjustments of parameters may be done to enhance the output.

\subsection{Feature based comparison of different eye gaze techniques}

As discussed above certain significant parameters on the basis of literature survey of the different eye gaze human interaction models have been identified for the study. Different eye gaze models have been selected, analysed and are summarized in Table 1.

The table is categorized into different categories for the classification of different features. These are the distance between the subject and the screen, different types of detection methods, the light source, the types of trackers, the position and movement of the head, capturing device and the visual angle. As discussed above following detections methods have been considered in this study. The appearance based methods $[15,27-29,33,40]$ may include pupil and purkinje or pupil and glint vector for eye detection. Shape based methods $[9,20,27,32,35]$ may use iris and corner detection, corneal reflection or pupil, purkinje and glint etc.

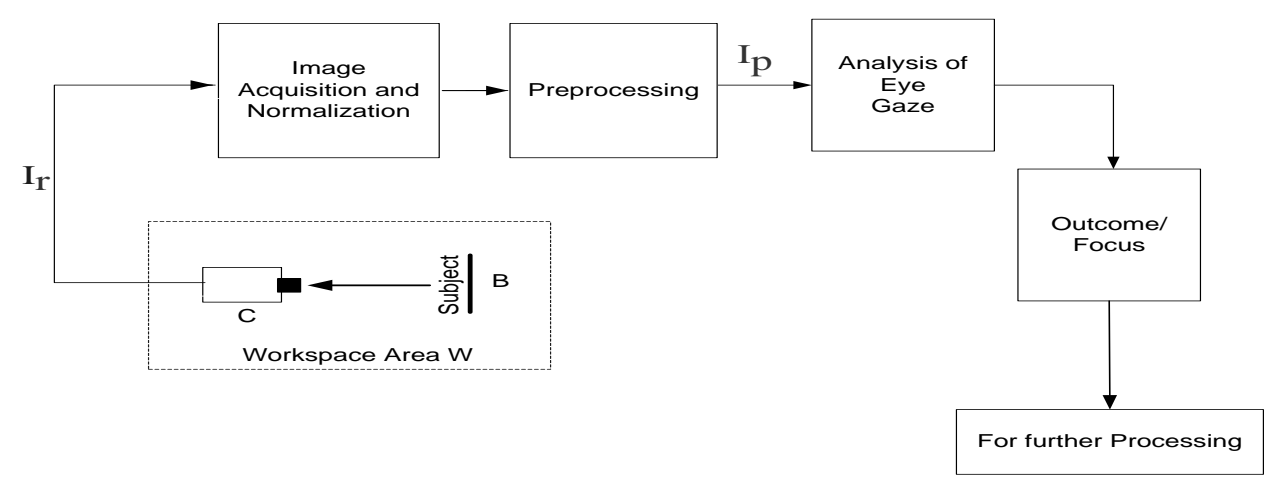

Fig 1: Schematic Diagram showing the overview of Eye Gaze Processing 
Feature based methods $[5,29,43]$ can have any one of the methods like pupil detection, corneal reflection etc. Feature based methods show better performance in indoors as well as in dark than in outdoors due to the shrinking of the pupil.

Template based methods $[21,22]$ use deformable template or between the eyes methods [8]. The other methods can use combination of the two or some of the above mentioned methods. PCCR and optic axis are also used in eye detection [27]. Similarly another detection method [29] involves the estimation of the eye gaze using the edge corners, center of the eye socket and the eye pupil. Such system does not require any wearable attachments, supplementary lighting and no use of the eye-glint phenomena. Another detection method [8] is not affected by the beard or moustache. It works, even if the low half of the face is hidden. Border detection [22] is one of the methods being used. EOG [14] is also in practice although not that much common due to drifting of the EOG's signal baseline.

The distance is also one of the significant factors and the distance column in the table is divided into three categories ranging from distance less than $60 \mathrm{~cm}$, between 60 to $100 \mathrm{~cm}$ and greater than $100 \mathrm{~cm}$. Experiments shows that when the distance between the user and the screen is long then the captured eye size is too small to detect. For accurate gaze tracking longer focal length camera can be used. Low cost, long range and long distance eye tracker is used with the free head movement. The PoG correction technique show comparable accuracy to desktop systems. A slight lower accuracy is resulted when the viewer moved a large distance [41]. The distance is $60 \mathrm{~cm}$ for normal participants and $300 \mathrm{~cm}$ for ALS user [28]. The Light column indicates under which condition the method operates like indoors (I), outdoors (O), with one IR light (IR), or IR with LEDs (IRL). The number of lights also affects the accuracy of the captured eye. The accuracy changes with the light conditions and is lower in outdoors [9]. Feature based methods show better performance in indoors as well as in dark than in outdoors due to pupil shrinking. The diagnostic and interactive trackers use different ways to detect eye. First one uses VOG with PoG and other observe the gaze behaviour. The interactive trackers has stereo-camera based gaze tracking system. The head pose is also one of the significant factors while detecting eye gaze. The head position can be fixed or free to some degree. The system may use a single camera or multiple cameras which may emit the light which can be IR light or visible or passive light. It is used particularly in all stages of eye detection and tracking. Although using multiple cameras like narrow and wide angle camera is more expensive but the accuracy is higher and enhanced. The visual angle in the table is the angle that a viewed object subtends at the eye, usually stated in degrees of arc. It also is called the object's angular size. The systems with lower visual angle accuracy are stronger in terms of selection of targets and it is used for benchmarking of the eye gaze systems [22]. The result and discussion presented here shows that there are significant phases for processing of eye gaze. Understanding of these phases helps to improve, enhance and optimize the efficiency of eye gaze models and algorithms.

The analysis of the algorithms on the basis of selected technical features as discussed has been presented and the results have been discussed.

This analysis is helpful to understand the various techniques used in the different eye gaze models by the researchers.In addition different techniques and methods which will affect the efficiency of eye gaze models have also been categorized.

\section{CONCLUSION}

Eye gaze techniques are being used to develop more intuitive and natural human interfaces with eye gaze techniques. There are various real time and other high end applications of eye gaze techniques in the field of sports, expression or emotion recognition, ophthalmology, neuroscience, medical research, driver's behaviour, shopper's and advertising research. The eye gaze techniques can be categorized into different detection methods like appearance, shape, template and feature, regression or 3D based. These methods may use pupil, eyelids, iris, glints, eye corners etc. Different types of trackers and techniques or algorithms are being used for eye detection. Further it has been observed that the accuracy of the eye gaze models depends on the several factors like visual angle, light source, head movements, determination of iris radius, center of the pupil and center of the cornea, distance between pupil and cornea, edge corners of the

eye region, the center of the eye socket and the eyepupil, distance of the subject from the camera, pupil size, lens, eye motions, eyelid motions, camera motions etc.

Careful selection of the certain parameters, controlling and adjustments of selected parameters is being done to enhance the output.These factors may further be used to design low cost systems with optimal use of available resources.Further it is desirable to use existing available resources, maximizing the extent of visual angle, reducing the calibration and complexity of the algorithms.This study has been conducted with two objectives. Firstly an overview of eye gaze process has been presented which includes various important phases for analysis and detection of the eye gaze. Secondly a feature based comparison of different eye gaze techniques has been presented.These features have been selected on the basis of extensive research survey. The features having more relevance and impact on the outcome of the eye gaze techniques have been identified.An analysis and classification of various eye gaze techniques, models and algorithms on the basis of selected parameters helps to understand the working and the design of the eye gaze models. This shall further help to optimize, improve or suggests certain enhancements for improving the performance of eye gaze techniques.

\section{FUTURE WORK}

The future study shall be on the selection of more parameters on the basis of further analysis of different algorithms or models. Further investigation shall be carried out to understand the effect of various control parameters on the eye gaze techniques in order to optimize and enhance the performance of the eye gaze model. 
Table 1. Feature based comparison of different eye gaze techniques based on significant factors

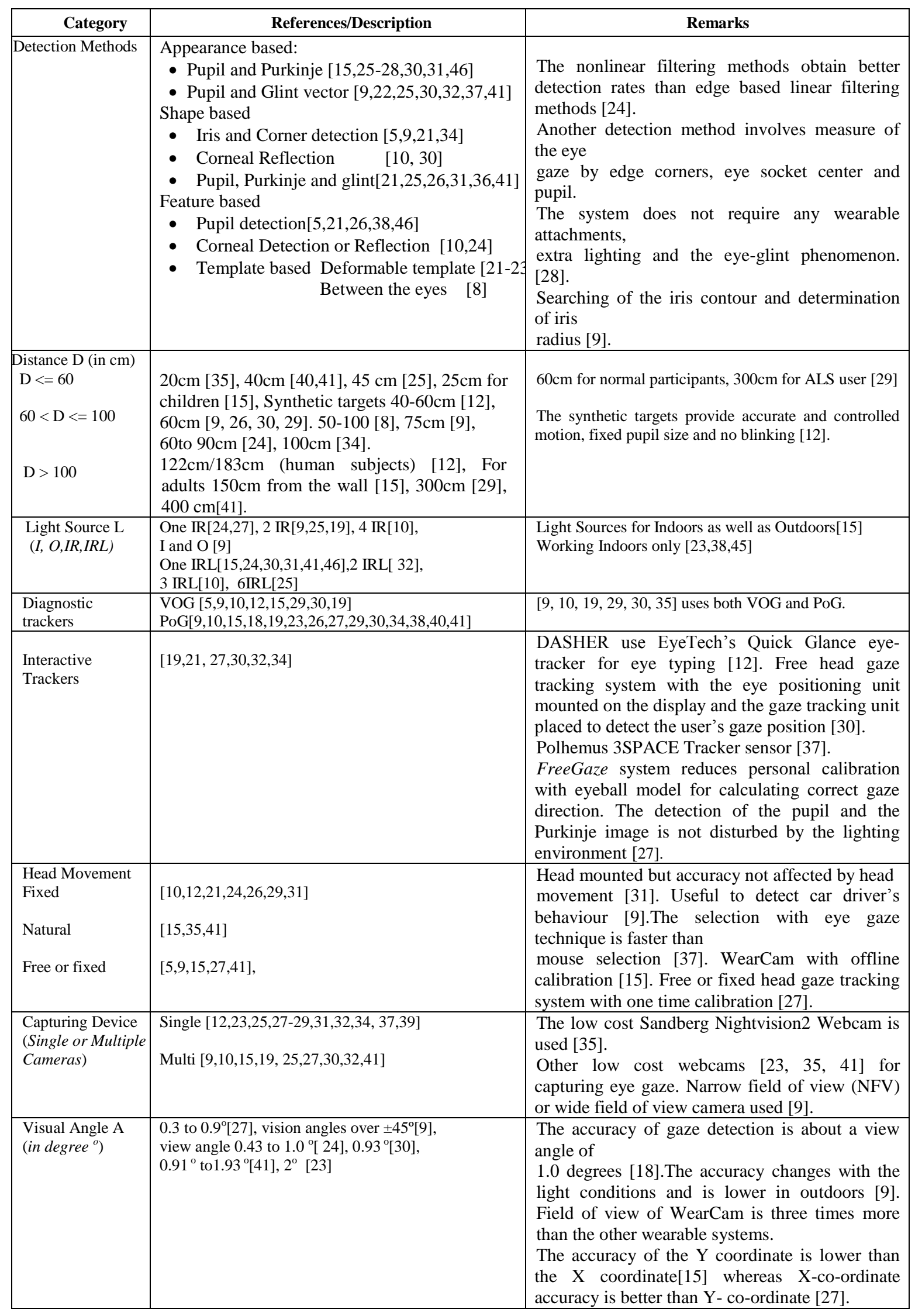




\section{REFERENCES}

[1] Hewett, Baecker, Card, Carey, Gasen, Mantei, Perlman, Strong, and Verplank. 1992. HumanComputer Interaction, In ACM SIGCHI Curricula for Human-Computer Interaction, Chapter 2, 5-6.

[2] Diaper, D. (2004). The Handbook of Task Analysis for Human-Computer Interaction. Lawrence Erlbaum Associates Inc., 393.

[3] Zhang, P., Benbasat, I., Carey, J. Davis, F., Galletta, D. and Strong, D. 2002. Human-computer interaction research in the MIS discipline. Comm. of the AIS, 9, 20, 334-355.

[4] Orman, Z., Abdulkadir, B. and Kemer, D. 2011. A Study on face, eye detection and gaze estimation. IJCSES, 2, 3, 29-46.

[5] Corcoran, P., Nanu, F., Petrescu, S. and Bigioi, P. 2012. Real-Time Eye Gaze Tracking for Gaming Design and Consumer Electronics Systems. IEEE Trans. On Consumer Electronics, 58, 2, 347-355, IEEE.

[6] Erol, A., Bebis, G., Nicolescu, M., Boyle, R. D., and Twombly, X. 2007. Vision-based hands pose estimation: A review. Computer Vision and Image Understanding, 108, 52-73, Elsevier Inc.

[7] Kinoshita, K., Ma, Y., Lao, S., and Kawade, M. 2006. A Fast and Robust 3D Head Pose and Gaze Estimation System. ICMI, ACM.

[8] Kawato, S. and Tetsutani, N. 2004. Detection and tracking of eyes for gaze-camera control. Image and Vision Computing, 22, 1031-1038, Elsevier.

[9] Perez, A., Cordoba, M. L., Garcia, A., Mendez, R., Munoz, M. L., Pedraza, J. L., and Sanchez, F. A. 2003. Precise eye gaze detection and tracking system. In Proceedings. Of WSCG Posters.

[10] Wilson, M., McGrath, J., Vine, S., Brewer, J., Defriend, D., and Masters, R. 2011. Perceptual impairment and psychomotor control in virtual laparoscopic surgery. Surg. Endo., Springer, 25, 2268-2274.

[11] Shy $u$, K. K.., Lee, P. L., Lee, M. H., Lin, M. H., Lai, R. J., and Chi $u$, Y. J. 2010. Development of a Low-Cost FPGA Based SSVEP BCI Multimedia Control System. IEEE Trans. on Biomedical Circuits and Systems, 4, 2, 125-132.

[12] Amir, A., Zimet, L. Sangiovanni, A. V., and Kao, S. 2005. An Embedded System for An EyeDetection Sensor. Computer Vision and Image Understanding, 98, 104-123, Published by Elsevier Inc.

[13] Mantiuk, R., Kowalik, M., Nowosielski, A., and Bazyluk, B. 2012. Do-It-Yourself Eye Tracker: Low-Cost Pupil Based Eye Tracker for Computer Graphics Applications. MMM, Springer-Verlag, 7131, 115-125.

[14] Yagi, T. 2010. Eye-gaze Interfaces using Electrooculography (EOG), EGIHMI, ACM.

[15] Noris, B., Keller, J. B, and Billard, A. 2011. A wearable gaze tracking system for children in unconstrained environments. Computer Vision and Image Understanding, 115, 476-486, Published by Elsevier Inc.

[16] Eye Tracking Research. Internet: http://www.tobii.com/en/eye-trackingresearch/global/research, [Jan. 10, 2013].

[17] Hansen, D. W. and Ji, Q. 2010. In the Eye of the Beholder: A Survey of Models for Eyes and Gaze. 32, 3, 478-500, IEEE Computer Society.

[18] Ward, D. and MacKay, D. 2002. Fast Hands-Free Writing By Gaze Direction. Nature Publishing Group, 418, 838.

[19] Kim, K. and Ramakrishna, R.S. 1999. VisionBased Eye-Gaze Tracking for Human Computer Interface. In Proceedings of IEEE Systems, Man and Cybernetics Conference, 2, 324-329.

[20] Poole, A. and Ball, L. J. 2005. Eye Tracking in Human-Computer Interaction and Usability Research: Current Status and Future Prospects. Encyclopedia of Human-Computer Interaction, Idea Group Inc.

[21] D. Kim, J. Jung, T.T. Nguyen, D. Kim, M. Kim, K.H. Kwon, and J.W. Jeon, " An FPGA-based Parallel Hardware Architecture for Real-time Eye Detection," Journal of Semiconductor Technology and Science, 12, 2, 150-161, 2012.

[22] Xie, X., Sudhakar, R., and Zhuang, H. 1994. On Improving Eye Feature-Extraction Using Deformable Templates. Pattern Recognition, 27, 6 , 791-799.

[23] I. F. Ince and J. W. Kim. "A 2D Eye Gaze Estimation System With Low Resolution Webcam Images," EURASIP Journal on Advances in Signal Processing. [Online]. 1-11.Available: http://asp.eurasipjournals.com/content/2011/1/40. [Sep. 4, 2012].

[24] S. A. Sirohey and A. Rosenfeld, "Eye Detection in a Face Image Using Linear and Nonlinear Filters," Journal of the Pattern Recognition Society, 34, 1367-1391, Elsevir, 2001

[25] Morency, L. P., Christoudias, C. M., and Darrell, T. 2006. Recognizing Gaze Aversion Gestures in Embodied Conversational Discourse. ICMI, ACM.

[26] Robinson, D. A. 1963. A method of measuring eye movements using a scleral search coil in a magnetic field. IEEE Trans. Biomedical Eng., 10, 137-145.

[27] Ohno, T., Mukawa, N., and Yoshikawa, A. 2002. Freegaze: A Gaze Tracking system for everyday Gaze Interaction,” In proceedings of Eye Tracking Research Applications Sym., 125-132.

[28] Shih, S. W., Wu, Y. T., and Liu, J. 2000. A Calibration-Free Gaze Tracking Technique. In Proceedings of the 15th Int'l Conference on Pattern Recognition (ICPR), 201-204.

[29] Agustin, J. S., Skovsgaard, H., Barret, M., Tall, M., Hansen, D. W., and Hansen, J. P. 2010. Evaluation of a Low-Cost Open-Source Gaze Tracker. 77-80, ACM, ETRA. 
[30] Ohno, T. and Mukawa, N. 2004. A Free-head, Simple Calibration, Gaze Tracking System That Enables Gaze Based Interaction. ETRA, ACM, 115-122.

[31] Viola, P. and Jones, M. 2001. Robust Real-Time Face Detection. In Proceedings of Eighth IEEE Int'l Conf. Computer Vision, 2, 747.

[32] Pentland, A., Moghaddam, B., Starner. T., and Turk, M. 1994. Viewbased and modular eigenspaces for face recognition. In Proceedings of IEEE Computer Society Conference on Computer Vision and Pattern Recognition, 84-91.

[33] Herpers, R., Michaelis, M., Lichtenauer, K. H., and Sommer, G. 1996. Edge and Keypoint Detection in Facial Regions. In Proceedings of Second International Conference on Automatic Face and Gesture Recognition, 212-217.

[34] C. Choo, J. W. Lee, K.Y. Shin, E. C. Lee, K. R. Park, H. Lee, and J. Cha. 2012. Gaze Detection by Wearable Eye-Tracking and NIR LED-Based Head-Tracking Device Based on SVR. ETRI Journal, 34, 4, 542-552.

[35] Zhu, Z. and Ji, Q. 2007. Novel Eye Gaze Tracking Techniques Under Natural Head Movement. IEEE Trans. on biomedical engineering, 54, 12, 22462260.

[36] Buscher, G., Biedert, R., Heinesch, D., and Dengel, R. 2010. Eye Tracking Analysis of Preferred Reading Regions on the Screen. CHI, ACM.

[37] Sibert, L. E. and Jacob, R. J. K. 2000. Evaluation of Eye Gaze Interaction. In Proceedings of the Computer Human Interaction, 281-288, ACM, http://citeseer.nj.nec.com/article/sibert00evaluation .html.
[38] Urbina M. and Huckauf, A. 2010. Alternatives to Single Character Entry and Dwell Time Selection on Eye Typing. ETRA, ACM, 315-322.

[39] Yamazoe, H., Utsumi, A., Tomoko, Y., and Shinji, A. 2008. Remote Gaze Estimation with a Single Camera Based on Facial-Feature Tracking without Special Calibration Actions. In Proceedings of Sym. Eye Tracking Research and Applications, 245-250, ACM.

[40] S. D'Mello, A. Olney, C. Williams, and P. Hays, "Gaze tutor: A gaze-reactive intelligent tutoring system," International Journal of HumanComputer Studies. [Online]. pp. 377-398 Available: http://www.sciencedirect.com/science/article/pii/S 1071581912000250, 70, 5, 2012

[41] Hennessey, C. and Fiset, J. 2012. Long Range Eye Tracking: Bringing Eye Tracking into the Living Room. ETRA, ACM Press.

[42] Wang, J. G., Sung, E., and Venkateswarlua, R. 2005. Estimating The Eye Gaze From One Eye. Computer Vision and Image Understanding, 98, 1, 83-103.

[43] Villani, N. A., Beni, G., and White, J. 2008. EMOES: Eye Motion and Ocular Expression Simulator. World Academy of Science, Engineering and Technology, 22.

[44] Vivero, V., Barreira, N., Penedo, M. G., Cabrero, D., and Remeseiro, B. 2010. Directional Gaze Analysis in Webcam Video Sequences. SpringerVerlag, 316-324, ICIAR.

[45] Zhu, Z., Fujimura, K., and Ji, Q. 2002. Real-Time Eye Detection and Tracking under Various Light Conditions. In Proceedings of the Eye Tracking Research and Applications Sym., 139-144, ACM. 